De quelques enjeux et usages historiques du Français fondamental

\title{
Vocabulaires fondamentaux et Français fondamental : applications à l'apprentissage de l'orthographe
}

André Bandelier et Claude Cortier

\section{OpenEdition}

\section{Journals}

Édition électronique

URL : https://journals.openedition.org/dhfles/1202

DOI : $10.4000 /$ dhfles. 1202

ISSN : 2221-4038

Éditeur

Société Internationale pour l'Histoire du Français Langue Étrangère ou Seconde

Édition imprimée

Date de publication : 1 janvier 2006

Pagination : 139-142

ISSN : 0992-7654

Référence électronique

André Bandelier et Claude Cortier, « Vocabulaires fondamentaux et Français fondamental : applications à l'apprentissage de l'orthographe », Documents pour l'histoire du français langue étrangère ou seconde [En ligne], 36 | 2006, mis en ligne le 06 septembre 2011, consulté le 27 mai 2021. URL : http:// journals.openedition.org/dhfles/1202 ; DOI : https://doi.org/10.4000/dhfles.1202

Ce document a été généré automatiquement le 27 mai 2021.

(c) SIHFLES 


\title{
Vocabulaires fondamentaux et Français fondamental : applications à l'apprentissage de l'orthographe
}

\author{
André Bandelier et Claude Cortier
}

1 La recherche d'un français restreint, limité à des fins pédagogiques et didactiques n'est pas nouvelle. On en trouve écho dans les travaux des premiers congressistes de l'Alliance française - notamment en 1887 -où, plutôt que d'imposer une réforme orthographique à tous les usagers du français, certains avaient envisagé de constituer un ou plusieurs " français réduits » à destination des différentes colonies (Bulletin de l'Alliance française 44, Chevalier 1997).

2 On retrouve cette même volonté de constituer un vocabulaire fondamental, alliant souvent des critères de choix de composition et des degrés de difficulté orthographique, dans des travaux américains, suisses ou belges (Prescott 1929, Vander Becke 1935, Haygood 1936, Aristizabal 1938, Dubois 1939, Dubois-Buyse 1940, Dottrens 1946). Pour ces auteurs, concepteurs de vocabulaires de base ou d'échelles de progression orthographique en langue maternelle, « un bon vocabulaire d'orthographe doit puiser dans le vocabulaire des enfants» (Aristizabal 1938). C'est pourquoi ces vocabulaires seront souvent mixtes, constitués à partir de textes d'adultes et de rédactions d'enfants de différents âges et degrés (voir Suso Lopez, ici-même). On établit ensuite la gradation des mots pour l'apprentissage en fonction des fréquences d'emploi et du degré de difficulté orthographique du mot.

3 Cet article souhaite faire état de travaux plus récents qui permettront d'inscrire dans la continuité certains usages ou "dérives" - comme on l'a dit parfois - du Français fondamental dans l'enseignement du français et notamment son utilisation comme référence pour la construction de progressions orthographiques et grammaticales.

4 Dans les années 1970 à l'Université de Neuchâtel, la partie "Orthographe d'usage » d'un Cours d'orthographe française (première édition imprimée : Séminaire de français moderne 1980 ; éditions subséquentes corrigées : 1987, 1994, 1995, 1997) a été élaborée à partir du Dictionnaire fondamental de la langue française, de Georges Gougenheim (1958). 
Un peu plus tard, en 1975, c'est au Japon que Nancy Le Floch va élaborer pour l'Athénée français de Tokyo, une Initiation progressive à l'orthographe du français. Vocabulaire et grammaire du Français fondamental ler degré. La présentation de ces expériences permettra d'apporter un témoignage sur l'intérêt, si ce n'est la nécessité, pour les enseignants, qu'ils soient de français langue étrangère ou langue maternelle, de se référer à des outils rigoureusement ou scientifiquement élaborés pour la sélection des contenus linguistiques, et notamment pour l'initiation à la maîtrise de la graphie du français et de l'orthographe dite d'usage.

\section{1. "Un cours d'orthographe pour non-francophones » (témoignage d'André Bandelier)}

Nouvel enseignant au Séminaire de français moderne de l'Université de Neuchâtel (désormais SFM), j'ai été associé au début des années 1970 à la refonte de son fascicule d'orthographe. Dans une institution qui a fêté son centenaire en 1992 et qui a gardé jusqu'aujourd'hui sa tradition littéraire et scripturaire (Terrier 1997) ${ }^{1}$ celui-ci se présentait sous la forme classique : orthographe grammaticale avec large place ménagée à l'accord du participe passé ; homonymes grammaticaux; maitrise de l'accentuation graphique et autres signes diacritiques. Chargé de l'élaboration d'une partie complémentaire dite d'orthographe d'usage, il a été choisi de recourir alors au Dictionnaire fondamental de la langue française, dans des conditions particulières.

Le "Cours d'orthographe (niveau moyen) ", successeur du "Cours d'orthographe et dictée ", a finalement pris le titre de "Cours d'orthographe et vocabulaire d'usage ». Dispensé à trois niveaux (étudiants avancés, moyens, faibles) selon des méthodologies adaptées, il préparait toutefois à une évaluation commune après deux semestres d'étude.

7 Le séminaire de français moderne fut pionnier en Suisse dans l'installation d'un laboratoire de langues. La création en 1965 du Centre de linguistique appliquée lui a apporté, davantage qu'un renouvellement de ses enseignements, un appui dans ceux de l'oral. A cheval sur les deux institutions, l'ancien instituteur que j'étais, qui (pour caricaturer) était sorti de sa formation première avec la conviction qu'il n'existait que cinq voyelles en français, a rapidement bénéficié des apports du nouvel institut. Je profitais des acquis récents des collègues : retombées indirectes des initiations du CREDIF pour l'utilisation des méthodes audiovisuelles au Cours de vacances, et des colloques scientifiques internationaux, dans une perspective plus large apportée en particulier par Georges-Eddy Roulet (Bandelier 1977). La présence de la linguistique statistique s'imposait par des bandes de phonétique corrective ${ }^{2}$ et des exercices structuraux de grammaire ajoutant doctement et invariablement après l'énoncé du contenu la mention "vocabulaire fondamental, premier degré». La présence en bibliothèque et l'état de l'ouvrage prouvent que les rédacteurs du matériel pédagogique recouraient systématiquement aux listes de fréquence de L'élaboration du français élémentaire (1956 : 63-109), ainsi qu'à sa version plus complète de 1964, L'élaboration du français fondamental (1er degré) (70-113).

8 Dès lors, on s'étonnera moins que j'aie spontanément essayé d'associer la maîtrise de la graphie du français avec les instruments destinés à la revalorisation de l'oral, le Français fondamental (FF) et le dictionnaire de G. Gougenheim. Ce dernier recourait lui- 
même aux deux premiers inventaires de fréquence disponibles en français, établis aux États-Unis dans l'entre-deux-guerres sur le dépouillement de textes écrits : les French Word Book, de V.A.C. Henmon (1924) et de G. E. Van der Beke (1935). Personnellement, j'avais été impressionné par l'intérêt que présentait, pour la mesure de la compréhension orale, un exercice alors décrié voire banni des classes, la dictée traditionnelle : «exercice imprudent, presque toujours inutile et fatalement dangereux... [qui] enseigne donc à faire des fautes » (Grandjouan $1970: 85$ ). Nous avions l'habitude de proposer un enseignement individualisé au laboratoire de langues fondé sur deux piliers : d'une part, la provenance des étudiants et les enseignements de la linguistique contrastive ; d'autre part, la passation de tests objectifs de discrimination auditive (Gilliard, Bandelier et Jeanneret 1971). Mais de simples dictées, tirées de coupures de journaux ou de périodiques, utilisées comme tests de répartition en classes plus ou moins homogènes au début de chaque semestre, offraient les multiples possibilités diagnostiques de la faute considérée positivement. Elles révélaient quasiment à tout coup la provenance linguistique. Reflets du passage obligé de la phonie à la graphie, les erreurs livraient, en sus des interférences facilement repérables avec les langues maternelles et des traces de stratégies d'apprentissage, des pistes pour la phonétique corrective individuelle que les tests objectifs n'avaient pas forcément révélées. Aussi est-ce en toute conscience de l'hétérodoxie de la démarche que j'ai voulu rapprocher des enseignements qu'on avait tendance à séparer. C'est dans le même état d'esprit que j'ai conçu plus tard des cours pratiques pour l'apprentissage et le perfectionnement de la rédaction, intitulés " De l'oral à l'écrit ». Je partais, entre autres, des scripts des enregistrements de L'élaboration du français fondamental (1er degré) (239-253).

9 Le Cours d'orthographe ronéotypé des années 1970 (Brunko-Méautis, Bandelier, Eigeldinger) comprend deux chapitres qui relevaient de ma compétence. Premièrement, le chapitre "Consonnes simples ou doubles». Ces douze pages, regroupées autour des différentes consonnes graphiques susceptibles de redoublement, se décomposent invariablement en trois parties: un pavé avec l'énoncé d'une règle synthétique; une liste de mots d'après Gougenheim ; une seconde série, réputée plus difficile, constituée par les enseignants. Chaque liste est accompagnée d'un ou deux exercices : la première s'appuie sur la consultation du Dictionnaire fondamental ; la seconde invite à ouvrir un dictionnaire de langue plus complet. Secondement, le chapitre « Finales ». Celui-ci regroupe sur trente pages, brièvement, les consonnes dites latentes (regroupement fondé donc à la fois sur la graphie et la phonie), plus complètement, les finales vocaliques et les finales consonantiques (basées sur le phonétisme). Les graphies diverses de chaque phonème sont illustrées par des mots du Français fondamental. Au besoin, on recourt à un vocabulaire plus étendu, dûment signalé, quand le champ des possibles n'est pas entièrement ou pas suffisamment couvert par le dictionnaire de Gougenheim. Les exercices mettent en action les règles lexicales de la composition et de la dérivation.

10 Selon le principe qui avait présidé au premier polycopié, les éditions successives du Cours d'orthographe française (Brunko-Méautis, Bandelier, Eigeldinger 1980 et 1987 ; Bandelier, Eigeldinger, Rytz, 1994, 1995 et 1997) présupposent l'analyse préalable du Dictionnaire fondamental (1958) et la répartition de ses entrées en fonction des critères retenus. L'évolution des imprimés peut être caractérisée de deux manières opposées. D'abord, les listes du Français fondamental ont été toujours plus largement utilisées. Sans pouvoir viser à l'exhaustivité des 3000 mots, on peut prétendre que le but principal, 
contrôler la maîtrise graphique et sémantique du Français fondamental, vérifié par les enseignants semaine après semaine durant deux semestres, a été atteint. 400 mots pour les consonnes simples ou doubles, 150 mots pour les consonnes et voyelles latentes (dans l'intervalle, on a adjoint le problème du e caduc), 850 mots pour les finales vocaliques, près de 600 mots pour les finales consonantiques et peu de mots qui se répètent dans les diverses catégories, donnent le total appréciable d'un peu moins de 2000 mots. On a veillé aussi à mieux délimiter ce qui est de l'ordre de la graphie et de la phonie, en recourant davantage à la transcription phonétique.

11 Mais ensuite la récupération par des collègues, pédagogues compétents de tendance plutôt conservatrice, qui ont accepté avec réticence le phénomène de la variation ou les rectifications de l'orthographe du français dans les années 1990, se marque par la suppression des exercices d'application qui faisaient fonctionner la langue. Les listes sont devenues objets de devoirs, de mémorisation. Pour favoriser l'autonomie de l'apprenant et renforcer ses capacités à l'autodidactisme, j'ai continué pourtant à présenter à mes étudiants la place de cette orthographe dite d'usage dans le système complexe de l'orthographe française (Catach 1973 : phonogrammes, morphogrammes, logogram-mes) et les principes qui avaient présidé à l'élaboration du Français fondamental (notions de fréquence, de répartition ou dispersion et de disponibilité). Seul, j'ai poursuivi le travail à partir des premiers exercices, en abandonnant quelque peu les recherches avec dictionnaire au profit de jeux linguistiques. Les dérivations, avec l'entraînement des mécanismes morphologiques et l'observation de régularités importantes comme la possibilité de repérer le genre de centaines de noms, sont conjuguées avec les transformations syntaxiques dans l'esprit du Livret méthodologique (Larousse 1971) qui accompagnait le Dictionnaire du français contemporain. Retraité depuis 2003, je garde l'impression d'avoir ainsi maintenu dans cette discipline l'exigence du contrôle des contenus, notamment par le recours au Français fondamental, et d'avoir donné la préférence à l'activité la plus naturelle possible par le recours à la linguistique structurale et générative.

\section{Mise en perspective (note de Claude Cortier)}

Dans l'introduction à L'initiation progressive à l'orthographe du français, éditée au Japon en 1975, N. Le Floch souligne que la méthode a été établie en fonction du vocabulaire du Français fondamental 1er degré, que l'ordre d'introduction des mots, expressions et tournures suit celui de Voix et images de France, parce qu'il fournit « une progression simple, claire et logique, applicable quelle que soit la méthode employée pour enseigner le français ». Tout comme A. Bandelier, N. Le Floch souligne la nécessité d'initier très tôt les élèves à la transcription correcte des sons du français : « On ne peut guère imaginer de situation d'écriture sans prononciation implicite de ce que l'on écrit ", mais aussi la nécessité d'accoutumer les élèves à l'orthographe des mots les plus fréquents et notamment aux plus fréquents de tous, les mots grammaticaux. Elle recommande également de respecter le principe de n'aborder un mot sous sa forme écrite que lorsqu'il est connu dans sa forme orale. Le passage a l'écrit, qui dans cette méthode fait également appel à la dictée, a été conçu pour être relativement tardif, en fonction du contexte japonais, car il est indispensable d'assurer d'abord au maximum la correction phonétique des élèves. Il faut aussi pouvoir disposer d'un matériel linguistique (structures, lexique) suffisant pour éviter que les dictées et les exercices se 
limitent à une simple transcription de mots isolés. Les dictées sont composées à l'aide de mots appris et connus qui peuvent donc être transcrits par les étudiants qui ne connaissent que la graphie fondamentale des sons, il faut aussi faire appel à des mots qui apparaissent en acquisitions globales. Ces mots sont systématiquement des mots du Français fondamental. Ils pourront ultérieurement servir de base pour l'étude d'autres sons.

Comme on peut le constater, ces méthodes souhaitent exploiter la relation entre la fréquence de l'usage (oral et écrit) et la rapidité des acquisitions. Comme le soulignait déjà François Ters ${ }^{3}$ en 1955, «les enseignants peuvent et doivent dès à présent tirer parti de l'admirable instrument qu'est le Français fondamental pour qui veut enfin trouver une boussole pour se diriger intelligemment dans l'énorme chaos de l'enseignement du français ». La liste du Français élémentaire recouvre, dans les conditions normales d'une épreuve de dictée du certificat d'études, $75 \%$ des mots de la dictée et correspond, en référence à l'échelle Dubois-Buyse d'orthographe d'usage, à ce qui peut être acquis par $75 \%$ des élèves de primaire à l'âge de 11 ans. C'est pourquoi, «du syllabaire aux manuels du Certificat d'Etudes, d'immenses simplifications sont à faire et rien ne sera clair dans les esprits et dans la langue tant que ce fonds commun ne sera pas assimilé. » (Ters $1955: 28$ ).

En dépit de la diversité des publics, on peut, semble-t-il, à travers ces quelques exemples faire le constat d'une étonnante similitude entre ces approches pédagogiques qui se sont appuyées, en France ou à l'étranger, sur les listes du Français fondamental, et faire état dans une histoire du Français fondamental de ces usages qui le réinscrivent dans la continuité des travaux sur les vocabulaires de base.

\section{BIBLIOGRAPHIE}

ARISTIZABAL, M. (1938), Détermination expérimentale du vocabulaire écrit pour servir à l'enregistrement de l'orthographe à l'école primaire. Louvain : Université de Louvain.

BANDELIER, A. (1977), «De la psychologie du comportement à la sociolinguistique: dix ans de linguistique appliquée à l'Université de Neuchâtel», Techniques d'instruction 4, pp. 6-11 (repris dans TRANEL 15, 1989, pp. 79-84).

BRUNKO-MÉAUTIS, A., BANDELIER, A., EIGELDINGER, F. (1980), Cours d'orthographe française, Neuchâtel, Séminaire de français moderne, (deuxième édition corrigée, 1987).

BANDELIER, A., EIGELDINGER, F. et RYTZ, F. (1994), Cours d'orthographe française, Séminaire de français moderne, troisième édition entièrement refondue, (quatrième édition révisée, 1995; cinquième édition révisée, 1997).

CATACH, N. (1973), L'orthographe, numéro spécial de Langue française 20, Paris : Larousse.

CHEVALIER, J.C. (1997), «Phonétique expérimentale et cours pour étrangers en France à la fin du $19 e$ siècle », Hammar, E. (éd), Documents SIHFLES 19, pp 158-165. 
DOHRENS, R. (1946), Cahiers de Pédagogie expérimentale et de psychologie de l'enfant 1, Genève : Delachaux et Niestlé.

DOTTRENS, R. et MASSARENTI, D. (1948), Vocabulaire fondamental du français. Neuchâtel : Delachaux et Niestlé.

DOTTRENS, R. et MAZARETTI, D. (1949), Cahiers de Pédagogie expérimentale et de psychologie de l'enfant 4, Genève : Delachaux et Niestlé.

DUBOIS, F. ET BUYSE, R. (1952), Échelle Dubois-Buyse. Bulletin de la Société Alfred Binet 405. (Originally published 1940)

GILLIARD, A., BANDELIER, A. et JEANNERET, R. (1971), « Le laboratoire des langues », Les sciences de l'éducation. Pour l'ère nouvelle 3, Université de Caen : CERSE, pp.64-87.

GOUGENHEIM, G. (1958), Dictionnaire fondamental de la langue française nouvelle édition revue et augmentée, Paris : Didier.

GOUGENHEIM, G., MICHEA, R., RIVENC, P. et SAUVAGEOT, A. (1956), L'élaboration du français élémentaire. Etude sur l'établissement d'un vocabulaire et d'une grammaire de base, Paris : Didier. GOUGENHEIM, G., MICHEA, R., RIVENC, P. et SAUVAGEOT, A. (1964), L'élaboration du français fondamental (1er degré). Etude sur l'établissement d'un vocabulaire et d'une grammaire de base, Paris : Didier.

GRANDJOUAN, J. (1970), Cent pages sur la linguistique et l'enseignement du français aux étrangers, Paris : Didier.

HAYGOOD, J. D. (1937), Le vocabulaire fondamental du français. Etude pratique sur l'enseignement des langues vivantes. Droz ( $2^{\circ}$ tirage).

HENMON, V. C. A. (1924), A French word book based on a count of 400,000 running words. Madison: University of Wisconsin, Bureau of Educational Research.

LAGANE, R. (dir.) (1971), Dictionnaire du français contemporain. Livret méthodologique, Paris : Larousse.

LE FLOCH, N. (1975), Initiation progressive à l'orthographe du français, Centre de recherches pédagogiques de l'Athénée français.

MICHEA, R. (1949), Vocabulaire de base et formation intellectuelle, Anvers : De Sikkel.

MICHEA, R. (1950), «Vocabulaire et culture», Les langues modernes 3B, Paris : Nathan.

MICHEA, R. (1952), « Le vocabulaire de base en France et à l'étranger » Les langues modernes 6, Paris : Nathan.

PRESCOTT, M. D. A. (1929), Vocabulaire des enfants et des manuels de lecture. Archives de Psychologie 83-84, pp. 225-274.

SUSO LOPEZ, J. (2006), « Vocabulaires logiques, vocabulaires simplifiés et Français élémentaire », Documents SIHFLES 36.

TERRIER, P. (1997), « 100 ans d'enseignement du français langue étrangère à l'Université de Neuchâtel (1892-1992)», Documents SIHFLES 20, pp. 127-140.

TERS, F. (1955), « Le français élémentaire », L'Education nationale 28, La pratique de la classe.

TERS, F., MAYER, G. et REICHENBACH, D. (1969), L'échelle Dubois-Buyse d'orthographe usuelle française. Neuchâtel : Messeiller.

VANDER BEKE, G. E. (1935), French word book. New York Macmillan. 


\section{ANNEXES}

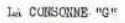

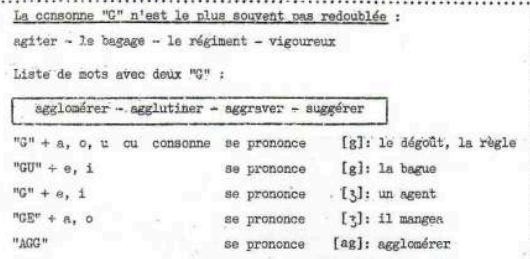

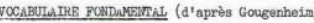

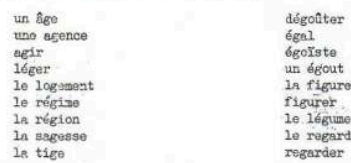

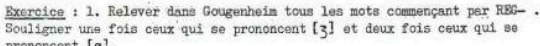
VOCABOLATES PLUS BTERO

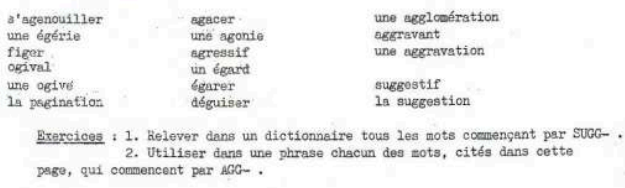

(Brunko, Bandelier, Eigeldinger, Cours d'orthographe,

Univerrité de Neuchàtel, SFTy, s.d.)

CONSONNES SIMPLES OU DOUBLE

LA CONSONNE *G *

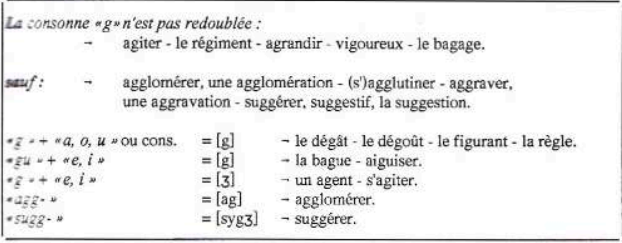

\begin{tabular}{|c|c|c|}
\hline $\begin{array}{l}{[3]} \\
\text { un âge } \\
\text { une agence } \\
\text { agir } \\
\text { forger }\end{array}$ & $\begin{array}{l}\text { léger } \\
\text { le logement } \\
\text { la nage } \\
\text { la neige }\end{array}$ & $\begin{array}{l}\text { le régime } \\
\text { la région } \\
\text { la sagesse } \\
\text { la tige }\end{array}$ \\
\hline $\begin{array}{l}\text { s'agenouiller } \\
\text { congeler } \\
\text { digeste }\end{array}$ & $\begin{array}{l}\text { figer } \\
\text { le logis } \\
\text { la magie }\end{array}$ & $\begin{array}{l}\text { ogival } \\
\text { une ogive } \\
\text { la pagination }\end{array}$ \\
\hline $\begin{array}{l}{[\mathrm{g}]} \\
\text { dègoûter } \\
\text { ègal } \\
\text { un égard }\end{array}$ & $\begin{array}{l}\text { égoiste } \\
\text { un égout } \\
\text { la figure } \\
\text { figurer }\end{array}$ & $\begin{array}{l}\text { le légume } \\
\text { un orgueil } \\
\text { regarder }\end{array}$ \\
\hline $\begin{array}{l}\text { agacer } \\
\text { une agonie } \\
\text { agressif }\end{array}$ & $\begin{array}{l}\text { déguiser } \\
\text { le dogme } \\
\text { un orgue }\end{array}$ & $\begin{array}{l}\text { régulariser } \\
\text { le sigle } \\
\text { la vogue }\end{array}$ \\
\hline
\end{tabular}

Principaux verbes en *-ger , qui ajoutent $* e * d e v a n t * a, o v$

corriger - elle corrige, vous corrigiez, mais - nous corrigeons, ils corrigeaient)

$\begin{array}{lll}\text { arranger } & \text { exiger } & \text { né } \text { liger } \\ \text { changer } & \text { juger } & \text { obliger } \\ \text { décourager } & \text { manger } & \text { partager } \\ \text { déranger } & \text { mélanger } & \text { plonger } \\ \text { diriger } & \text { nager } & \text { protéger }\end{array}$




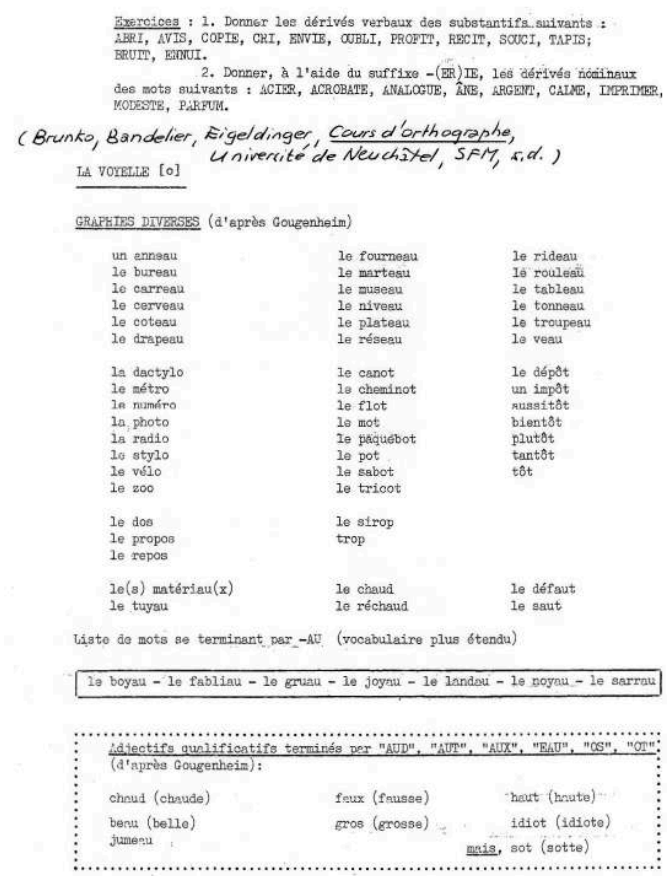

Bxorcicos : 1. Utiliser les homonymes sulvants dans des phrases différentes 2. Chorober à l'nide d'un dietionnaire las dérivgs des substen-

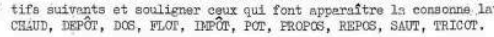

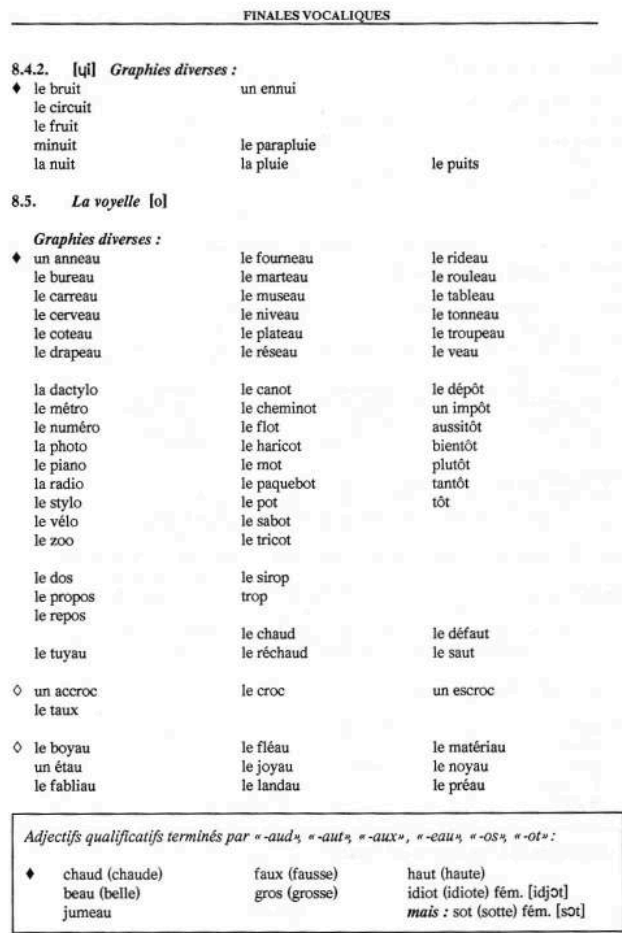

\section{NOTES}

1. Nouvelle appellation depuis le 1er octobre 2002 : Institut de langue et civilisation françaises (ILCF). 
2. Homologuées après une expertise, elles étaient diffusées par la Commission interuniversitaire suisse de linguistique appliquée (CILA).

3. François Ters sera auteur notamment de L'échelle Dubois-Buyse d'orthographe usuelle du français, OCDL, 1975, avec G. Mayer et D. Reichenbach. Il était alors professeur au Lycée Claude Bernard.

\section{RÉSUMÉS}

Les auteurs, A. Bandelier et C. Cortier, se fondent sur deux expériences pédagogiques des années 1970 dans l'enseignement du français langue étrangère ou seconde. Dans les deux cas, les enseignants ont utilisé le vocabulaire du Français fondamental. A l'Université de Neuchâtel, on a élaboré un Cours d'orthographe française, dont la partie dite d'orthographe d'usage visait principalement à la maîtrise graphique et sémantique des mots du Français fondamental; à l'Athénée français de Tokyo, N. Le Floch a produit L'initiation progressive à l'orthographe du français dans une perspective proche. L'expérience démontre l'intérêt pour les enseignants de se référer à des outils rigoureusement et scientifiquement élaborés pour la sélection des contenus linguistiques dans l'enseignement des langues.

The authors, A. Bandelier and C. Cortier, base their study on two pedagogical experiences of the 1970'in the field of teaching French as a second or foreign language. In the two cases, the teachers used the vocabulary of the "Fundamental French". At the University of Neuchâtel, a class of French orthography was elaborated, of which the section reffered to as "usual orthography" mainly aimed at the graphical and semantical mastery of the workes of the "Fundamental French"; at the French Athénée in Tokyo, N. Le Floch produced "The gradual initiation to French orthography" from a close angle. Experience shows the interest for the teachers to refer to scientifically and rigorously elaborated tools for the selection of the linguistic contents in the teaching of modern languages.

\section{INDEX}

Keywords : adult teaching, Basic vocabulary, French as a second/foreign language, Fundamental French, Gougenheim Georges

Mots-clés : Vocabulaires de base - Français fondamental - Gougenheim Georges - FLE Enseignement aux adultes

\section{AUTEURS}

\section{ANDRÉ BANDELIER}

Université de Neuchâtel

CLAUDE CORTIER

INRP 